\title{
Microwave transmissivity of a metamaterial-dielectric stack
}

\author{
Celia A. M. Butler, ${ }^{1, a)}$ James Parsons, ${ }^{1}$ J. Roy Sambles, ${ }^{1}$ Alastair P. Hibbins, ${ }^{1}$ and \\ Peter A. Hobson ${ }^{2}$ \\ ${ }^{1}$ Electromagnetic Materials Group, School of Physics, University of Exeter, Exeter EX4 4QL, \\ United Kingdom \\ ${ }^{2}$ QinetiQ, Cody Technology Park, Farnborough, Hampshire GU14 OLX, United Kingdom
}

(Received 24 July 2009; accepted 1 October 2009; published online 26 October 2009)

\begin{abstract}
A metamaterial layer comprising of a conducting square mesh surrounding subwavelength holes has a largely pure imaginary effective refractive index. We explore the microwave transmissivity of a stack of such metamaterial layers separated by dielectric spacers. As expected, a family of high transmissivity bands is experimentally observed. It is found that the lowest frequency edge is independent of the number of unit cells making up the structure and is highly tunable by appropriate geometrical design of the metamaterial layers. (C) 2009 American Institute of Physics.
\end{abstract}

[doi:10.1063/1.3253703]

Multilayer metal-dielectric structures have been extensively studied at visible frequencies, and their response utilized in areas such as electromagnetic shielding, nonlinear photonics and perfect lensing. ${ }^{1-3}$ Geffcken $^{4}$ in 1939 fabricated metal-dielectric thin film stacks that exhibited transmission features that were significantly narrower than those previously observed in conventional dielectric-dielectric multilayer arrangements. ${ }^{5}$

The spectral response of metal-dielectric stacks in the visible regime comprises of a series of photonic band gaps where the reflectivity is high (and the transmissivity is low), separated by a series of peaks of high transmissivity. These transmission peaks correspond to near-standing-wave resonances within each dielectric cavity, coupled together via exponential fields within the metal film. Near the high frequency band edge of the first transmission band, the electric fields are predominantly confined to the dielectric and pass through zero in the metal. In contrast, at the low frequency band edge a significant proportion of the field enhancement occurs inside the metal regions. ${ }^{6-8}$

An equivalent study of a metal-dielectric layer stack in the microwave domain $\left(10^{9}-10^{10} \mathrm{~Hz}\right)$ is at first sight impractical since the real and imaginary parts of the refractive index of metals are both large $\left(>10^{3}\right)$ and almost equal (i.e., the metal is near-perfectly conducting). Even a metal film of thickness $20 \mathrm{~nm}$ will almost completely screen the incident field ${ }^{9}$ because of the large impedance mismatch. Instead, a metal is structured on the subwavelength scale to create a metamaterial with effective electromagnetic properties which replicates the behavior of Drude-like (plasmonic) metals in the visible regime $\left(\mathrm{Ag}, \mathrm{Au}\right.$, etc.). ${ }^{10}$ The metamaterial layer consists of a non diffracting square metal mesh surrounding an array of identical square holes. At wavelengths greater than the size of the holes, the electromagnetic fields are exponential within the holes with a decay length that is primarily dictated by the metamaterial geometry. Consequently the metamaterial is equivalent to a thin layer with a pure imaginary refractive index. ${ }^{12,13}$

The sample shown in Fig. 1(a) comprises of eight printed circuit board (PCB) layers that are originally clad

${ }^{a)}$ Electronic mail: celia.butler@ex.ac.uk. with $18 \mu \mathrm{m}$ of copper on one face. The copper is removed from three of these substrates the remaining five being etched to leave a copper square mesh (Fig. 1(b)) with periodicity $\lambda_{\mathrm{g}}=5 \mathrm{~mm}$ and hole side length $w_{\mathrm{h}}=4.85 \mathrm{~mm}$. The PCB substrate is a low loss dielectric (Nelco NX9255) of thickness $t_{\mathrm{d}}=6.35 \mathrm{~mm}$, and relative permittivity $\sim 3$ across the studied frequency range. The sample is considered as infinite in the $x y$ plane since the sample area is much greater than the finite size of the microwave beam spot. A collimated plane wave $(5 \mathrm{GHz} \leq v \leq 30 \mathrm{GHz}$ ) is directed onto the $x y$ plane of the sample at normal incidence via a horn antenna placed at the focus of a spherical mirror. The transmitted radiation is then collected by a spherical mirror and focused into a detector horn. The experimental transmission spectrum (Fig. 2, circles) shows the first two transmitting bands. Note that the transmission resonances observed should not be confused with enhanced optical transmission (EOT) phenomena first recorded by Ebbesen et al., ${ }^{11}$ which rely on the excitation of diffractively coupled surface plasmons. More recently, Mary et al. have presented a theoretical analysis of the response of a double fishnet structure which combines the concept of EOT with diffractively coupled "spoof" surface-plasmons, ${ }^{14}$ and when stacked the response of their structure bear similarities to that presented here. However the frequency range used in the present study is far below the onset of diffraction at $60 \mathrm{GHz}$, and hence these surface plas-

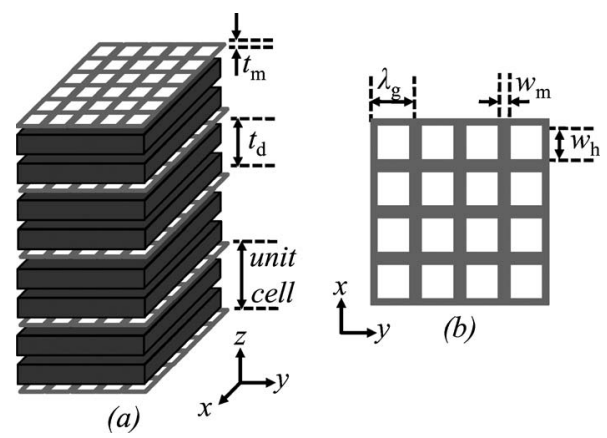

FIG. 1. (a) Exploded schematic of the metamaterial-dielectric experimental sample, dark regions represent the low loss dielectric Nelco NX9255 and light layers represent the metamaterial. (b) Plan view representation of the air-filled hole array metamaterial, where light gray represents copper. 


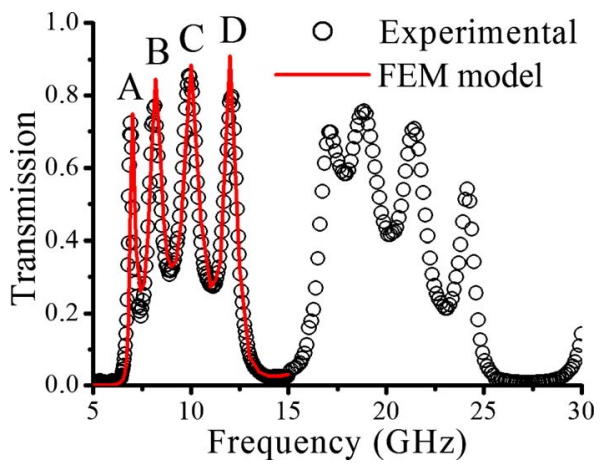

FIG. 2. (Color online) Experimental transmission spectra (circles) illustrating the first two pass bands separated by band gaps. Numerically modeled data are also shown (line) for the first pass band (5 GHz $\leq v \leq 15 \mathrm{GHz}$ ).

mon modes, spoof or otherwise, cannot play a role.

Each transmission band is comprised of a set of $N$ resonant modes, where $N$ is equal to the number of unit cells in the sample. In what follows, discussion is limited to the first transmission band since this is sufficient to fully understand the structure's fundamental behavior. (The resonances in bands at higher frequencies simply correspond to higher order modes in the dielectric cavities, retaining similar field distributions within the metamaterial.) The experimental transmission maxima relating to the four resonant modes in the first transmission band are labeled $A$ through $D$ in Fig. 2. A numerical prediction of the transmission spectra using a finite element method (FEM) mode ${ }^{15}$ is also shown (solid red line). Note to obtain an accurate prediction of the microwave response of the stack, an adequate representation of the fields within the very thin metamaterial layers is essential. This was achieved by increasing the density of the tetrahedral mesh employed in the model in the metamaterial regions until convergence was obtained. The dielectric permittivity of the PCB substrate used in the simulation was independently determined from experimentally measured complex reflection and transmission measurements to be $2.96 \leq \varepsilon$ $\leq 3.07$ and $0.001 \leq \tan \delta \leq 0.014$ over the frequency range of the lowest transmission band.

Figure 3 illustrates the predictions from the FEM model
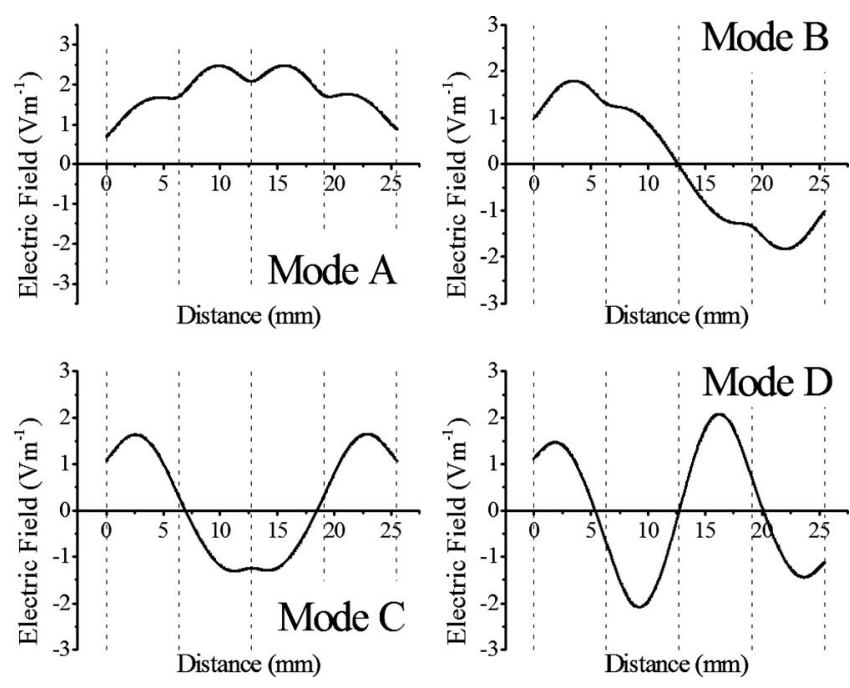

FIG. 3. Series of four graphs, labeled mode $A$ through mode $D$, representing the time-averaged electric field along a line plotted in the $z$ direction through the center of a hole for the four transmission maxima from Fig. 2. of the time-averaged electric field distributions along a line running in the $z$ direction passing through the center of the holes in the metamaterial layer. This modeling assumes that the meshes are all aligned. However it is worth noting that random translational misalignment of the meshes does not perturb the microwave response of the stack, since the decay length of the near fields (evanescent diffracted orders) associated with each metamaterial layer is approximately $1 \mathrm{~mm}$ or less at these frequencies, i.e., much smaller than the spacing of the metamaterial layers $\left(t_{\mathrm{d}}=6.35 \mathrm{~mm}\right)$. Of course if the thickness of the dielectric layer is reduced, or the periodicity of the array increased, then transverse mesh displacement may indeed perturb the response.

The transmission resonances observed (Fig. 2) occur when sine- or cosine-like standing-waves in the dielectric layers couple via exponential fields within the metamaterial layers. Our experimental geometry is similar to that recently studied analytically by Gadsdon et al. ${ }^{16}$ who considered alternating layers of untextured metal and dielectric films in the visible regime. They showed that the metal film acts as a tunnel barrier, with the fields within the metal films taking either a hyperbolic sine or hyperbolic cosine form. We propose that this is also the case for the metallic mesh in the metamaterial-dielectric multilayer stack studied here. The highest frequency mode $(D, 12.11 \mathrm{GHz})$ approximately defines the upper band edge (Fig. 2), and has an electric field in the metamaterial layer that is hyperbolic sinusoid in character, passing through zero. Therefore the fields in adjacent dielectric cavities oscillate out of phase, with the majority of the cavity oscillation being excluded from the metal (bottom right panel of Fig. 3). Hence the highest frequency mode $(D)$ is associated with the half-wavelength Fabry-Pérot condition ${ }^{17}$ for a single etalon, although slightly reduced in frequency due to the finite effective conductance (nonzero skin depth) of the metamaterial layer. However, of primary interest is the lowest frequency mode (mode $A, 6.93 \mathrm{GHz}$ ), whose field solution is shown in the top left panel of Fig. 3 . On resonance the electric fields in the dielectric cavities oscillate in phase with each other, since the exponential fields within the metamaterial layer take the form of a hyperbolic cosine. Now the wavelength of the mode within the dielectric cavity is much longer than simply twice the dielectric thickness because the hyperbolic cosine form of the field removes a significant proportion of the cavity oscillation from the dielectric. Importantly, this field solution is unlike that for an all dielectric multilayer stack. ${ }^{5}$ (Note that the hyperbolic parts of the field distribution do not occur symmetrically in the second and fourth metamaterial layers due to the impedance mismatch with free space at the top and bottom of the stack.) The consequence of this result is particularly striking when one considers the effect of an increase of the number of repeat units in the stack $^{16}$ from four to ten (see Fig. 4): the lowest frequency mode $(A)$ does not significantly move. For an all dielectric stack the lowest frequency mode would have a wavelength approximately twice the optical length of the overall structure and would linearly depend on the number of repeat units. Clearly the frequency of the mode that defines the upper band edge is not expected to alter when changing the number of repeat units due to the requirement that the electric field passes through zero in the metamaterial region in each unit cell. Thus the overall effect of the addition of extra cavities is only to increase the num- 


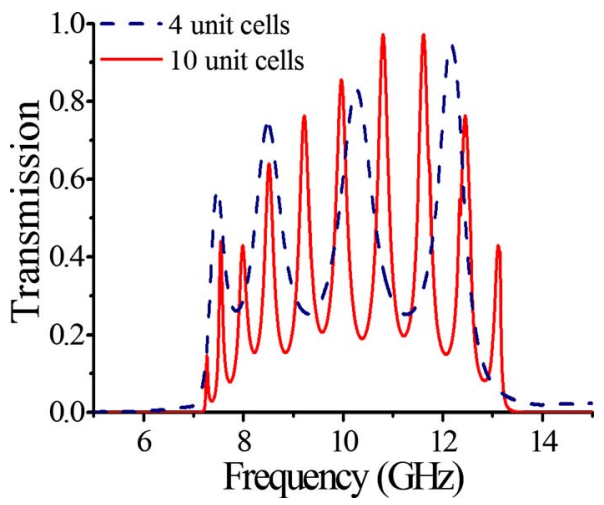

FIG. 4. (Color online) Transmission spectra for the first pass band region of two structures: the four unit cell stack (dashed line) and the ten unit cell stack (solid line), showing the band width does not significantly increase.

ber of modes within the bandwidth, which in turn is largely governed by the geometry of the unit cell. In this instance, there is no significant increase in the width of this band, only a sharpening of its edges. However any decrease in the hole size induces a decrease in the decay length of the evanescent fields into the metamaterial region, supporting a shorter effective wavelength in the dielectric cavity. This then alters significantly the frequency of the lowest mode (mode $A$ ) but does not shift that of the upper mode (mode $D$ ).

In summary, we have experimentally observed transmission bands in the microwave response of a metamaterialdielectric multilayer stack. Because the metamaterial has a largely imaginary effective refractive index, the frequency of the transmission band edges are mainly determined by the form of the exponential field distribution in these Drude-like metamaterial layers, which can take either a hyperbolic cosine or hyperbolic sine form. For the hyperbolic sine case (similar to the sine case for a dielectric), the fields within the metamaterial are small. Therefore the high frequency band edge is in close agreement to that expected for a single Fabry-Pérot etalon of the thickness of the dielectric, and has a very weak dependence on the overall length of the structure. However when the fields in the metamaterial are of hyperbolic cosine form a significant proportion of the cavity oscillation is removed from the dielectric and each dielectric cavity supports a standing-wave wavelength that is much longer than its optical length. Furthermore, the frequency of the lower band edge is independent of the number of unit cells but is highly tunable by appropriate design of the geometrical properties of the metamaterial layers. It is also interesting to note that if the thickness of the metamaterial layer is reduced (ultimately limited by the skin depth, $\sim 1 \mu \mathrm{m}$ ), the resonant wavelength of the low frequency mode will become larger than the thickness of the entire structure.

The authors wish to acknowledge the financial support of QinetiQ and the EPSRC (U.K.) for funding CAMB through the Industrial CASE scheme, and Great Western Research, in association with HP, for funding JP. APH also acknowledges the support of EPSRC through his Advanced Research Fellowship. The authors also wish to acknowledge C. R. Lawrence of QinetiQ for useful technical discussion.

${ }^{1}$ M. Scalora, M. J. Bloemer, and C. M. Bowden, J. Appl. Phys. 83, 2377 (1998).

${ }^{2}$ M. C. Larciprete, C. Sibilia, S. Paolini, and M. Bertolotti, J. Appl. Phys. 93, 5013 (2003).

${ }^{3}$ M. Scalora, M. J. Bloemer, and C. M. Bowden, Opt. Photonics News 10, 23 (1999)

${ }^{4}$ W. Geffcken, Deutsches Reich Patentschrift No. 716153 (8 December 1939).

${ }^{5}$ H. A. Macleod, Thin Film Optical Filters, 3rd ed. (Taylor \& Francis, London, 2001).

${ }^{6}$ M. Scalora, G. D’Aguanno, N. Mattiucci, M. J. Bloemer, D. de Ceglia, M. Centini, A. Mandatori, C. Sibilia, N. Akozbek, M. G. Cappeddu, M. Fowler, and J. W. Haus, Opt. Express 15, 508 (2007).

${ }^{7}$ W. Y. Zhang, X. Y. Lei, Z. L. Wang, D. G. Zheng, W. Y. Tam, C. T. Chan, and P. Sheng, Phys. Rev. Lett. 84, 2853 (2000).

${ }^{8}$ J. B. Pendry, Phys. Rev. Lett. 85, 3966 (2000).

${ }^{9}$ I. R. Hooper and J. R. Sambles, Opt. Express 16, 17258 (2008).

${ }^{10}$ J. B. Pendry, L. Martin-Moreno, and F. J. Garcia-Vidal, Science 305, 847 (2004).

${ }^{11}$ T. W. Ebbesen, H. J. Lezec, H. F. Ghaemi, T. Thio, and P. A. Wolff, Nature 391, 667 (1998).

${ }^{12}$ R. Sauleau, Ph. Coquet, J. P. Daniel, T. Matsui, and N. Hirose, Int. J. Infrared Millim. Waves 19, 1693 (1998).

${ }^{13}$ J. Bae, J-C. Chiao, K. Mizuno, and D. B. Rutledge, Int. J. Infrared Millim. Waves 16, 377 (1995).

${ }^{14}$ A. Mary, S. G. Rodrigo, L. Martin-Moreno, and F. J. Garcia-Vidal, J. Phys.: Condens. Matter 20, 304215 (2008).

${ }^{15}$ HFSS v.11, Ansoft Corporation, www.ansoft.com.

${ }^{16}$ M. R. Gadsdon, J. Parsons, and J. R. Sambles, J. Opt. Soc. Am. B 26, 734 (2009).

${ }^{17}$ C. Fabry and A. Pérot, Ann. Chim. Phys. 16, 115 (1899). 